\title{
La sostenibilidad: una mirada hacia las principales herramientas de evaluación en los destinos turísticos
}

DOI: https://doi.org/10.33262/ap.v1i3.3

\begin{abstract}
(c) (1) (2)(2)
Sustainability: a look at the main evaluation tools in tourist destinations
Martha Omara Robert Beatón, ${ }^{1}$ Maité Echarri Chávez, ${ }^{2}$ Jennifer Díaz Saraiba, ${ }^{3}$ \& Víctor Martínez Robert. ${ }^{4}$

\begin{abstract}
.
The present work has as objective to determine the tools, methods and models that allow to evaluate the sustainability in a tourist destination, taking as a base those referred by the scientific literature consulted and from parameters defined in previous research on the study of sustainability. Using fundamentally the synthesis methods of the bibliography on the subject matter of the study and the comparative analysis. The results of this analysis showed that the models and methods tools do not allow to evaluate the sustainability from an interdimentional, intradimentional and multidimensional approach that integrates the complex dynamics that concur in the management of a tourist destination, setting the bases to carry out investigations from other areas of the knowledge in the search for instruments aimed at recognizing and understanding the holistic vision of sustainability in tourist destinations.
\end{abstract}

Keywords: Sustainability, Evaluation, Tourist Destinations.

\section{Resumen.}

${ }^{1}$ Universidad de La Habana. Facultad de Turismo. La Habana, Cuba. omara.robert73@gmail.com.

${ }^{2}$ Universidad de La Habana. Facultad de Turismo. La Habana, Cuba. maite_echarri@ftur.uh.cu

3 Especialista Departamento Comercial. Agencia de Viajes San Cristóbal, La Habana, Cuba. omara.robert73@gmail.com.

${ }^{4}$ Universidad de La Habana. Facultad de Turismo. La Habana, Cuba. viticomartinez98@gmail.com. 
El presente trabajo tiene como objetivo determinar las herramientas, métodos y modelos que permitan evaluar la sostenibilidad en un destino turístico, tomando como base las que refiere la literatura científica consultada y a partir de parámetros definidos en investigaciones precedentes sobre el estudio de la sostenibilidad. Utilizando fundamentalmente los métodos de síntesis de la bibliografía sobre el tema objeto de estudio y el análisis comparativo. Los resultados de este análisis demostraron que las herramientas modelos y métodos no permiten evaluar la sostenibilidad desde un enfoque interdimensional, intradimensional y multidimensional que integre las dinámicas complejas que concurren en la gestión de un destino turístico, sentando las bases para realizar investigaciones desde otras áreas del conocimiento en la búsqueda de instrumentos dirigidos a reconocer y a comprender la visión holística de la sostenibilidad en los destinos turísticos.

Palabras claves: Sostenibilidad, Evaluación, Destinos Turísticos.

\section{Introducción.}

Después de la conferencia de Río de 1992, se debate de qué forma el turismo debe desempeñar un papel dentro del de desarrollo sostenible. En 1995 se elabora la Carta de Lanzarote, en el marco de la Conferencia Mundial de Turismo Sostenible y en 1996 la Agenda 21 para la industria de viajes y turismo.

En años posteriores se profundiza tanto en los diferentes ámbitos de la sostenibilidad aplicada el turismo como en los distintos niveles de actuación por los diferentes agentes. Alcanzando en el 2015 una agenda de desarrollo sostenible universal y transformadora, donde se ponen de manifiesto la experiencia de la comunidad internacional en materia de desarrollo de los últimos 20 años y se definen 17 objetivos (hasta el 2030) concretos y concisos en la búsqueda de un cambio de paradigma para las personas y el planeta; de ellos cinco responden específicamente a la actividad turística con el fin de mitigar las consecuencias de los modelos de desarrollo establecidos; declarando el 2017, "Año Internacional del Turismo Sostenible”.

Los estudios más recientes sobre sostenibilidad en el mundo han tratado la creación de herramientas para medir y/o evaluar varios aspectos de la sostenibilidad en el turismo (Palomeque et al., 2018; Torres, 2016; Vera et al., 2013; Velasco, 2011; Ávila \& Barrado, 2005). La Organización Mundial del Turismo (OMT) (1998, 2005, 2007) ha emitido varias guías para la evaluación del desarrollo sostenible en este sector. En el año 2005 esta organización emitió una guía práctica (lista de indicadores) para contribuir a erradicar problemas con respecto a la gestión del desarrollo sostenible en destinos turísticos.

En Cuba la mayoría de los estudios sobre sostenibilidad turística de destinos han sido realizados a partir de la determinación y evaluación de indicadores divididos de acuerdo a las dimensiones de la sostenibilidad. Esto ha estado determinado por la elaboración de la propuesta de indicadores de sostenibilidad para el turismo en Cuba, documento elaborado de 
conjunto entre el Ministerio de Turismo (MINTUR) y el Ministerio de Ciencia Tecnología y Medio Ambiente (CITMA) cubanos en el año 2003, el cual, aunque todavía insuficiente, ha constituido pauta para el desarrollo de las investigaciones más recientes con respecto a este tema en el país.

Entre esas investigaciones se encuentran las realizadas por Robert y Blanco (2016) para el destino Cuba, Echarri y Delis (2015) en el Centro Histórico de La Habana, sobre varios destinos de naturaleza cubanos. Estos estudios, aunque han coincidido en el método o herramienta a utilizar, difieren, al igual que los estudios internacionales sobre este tema, en cuanto al enfoque aplicado, así como en el proceso de determinación de los indicadores y, por supuesto, en los indicadores determinados para cada caso. (Robert y Blanco, 2016; Echarri y Delis, 2015).

Ante la multiplicidad de enfoques, modelos y herramientas, así como las diferencias entre las investigaciones realizadas siguiendo los mismos métodos para evaluar la sostenibilidad del desarrollo de los destinos turísticos, se hace necesario establecer una base uniforme para definir niveles de sostenibilidad y al mismo tiempo permita establecer comparaciones espaciales y temporales entre diferentes destinos turísticos, por lo que se plantea como objetivo de la presente investigación: Determinar herramienta, métodos y/o modelos que permitan evaluar la sostenibilidad en un destino turístico.

\section{Marco Teórico Referencial.}

El Informe Brundtland ${ }^{5}$, también conocido como Nuestro Futuro Común, estableció las bases para promover el desarrollo sostenible, en la búsqueda de la integridad ecológica, la eficiencia económica y la equidad social. La viabilidad y la eficiencia económica deben ser buscadas por medio del prudente crecimiento de la economía limitando la capacidad de carga del medio natural del territorio, o sea, un crecimiento a medida para cada caso.

La equidad social requiere la búsqueda de mejores indicadores de calidad de vida para las poblaciones menos favorecidas y la integridad ecológica hace referencia a la conservación de la biodiversidad de los recursos naturales (ONU, 1987).

El evento que marcó el inicio del cambio de perspectiva en relación con la actividad turística fue la realización de Globe '90 en Vancouver, Canadá, a comienzos de la década de 1990. Fue una gran conferencia internacional que se transformó en el primer foro de discusión sobre el desarrollo del turismo con base sostenible y reunió a investigadores, funcionarios públicos, organizaciones no gubernamentales y a otros sectores de la sociedad. En aquella ocasión, juntamente con la aprobación de diversas recomendaciones para lograr que el desarrollo del

\footnotetext{
${ }^{5}$ Es un informe que enfrenta y contrasta la postura de desarrollo económico actual junto con el de sostenibilidad ambiental, realizado por la ex-primera ministra de Noruega Gro Harlem Brundtland, con el propósito de analizar, criticar y replantear las políticas de desarrollo económico globalizador, reconociendo que el actual avance social se está llevando a cabo a un costo medioambiental alto.
} 
turismo fuese más sostenible, se hizo referencia a los beneficios que podrían resultar del mismo (Silveira, 2005).

La sostenibilidad pasa a ser un concepto central que propone la reevaluación del papel del turismo en la sociedad contemporánea. Concepto que demanda una visión a largo plazo de la actividad económica y que pretende ofrecer una respuesta al proceso de degradación ambiental del planeta y, también, a los problemas y desigualdades sociales generados por un estilo de crecimiento económico que, además de comprometer la satisfacción de las necesidades de grandes sectores de la población mundial limita las oportunidades de las generaciones futuras para satisfacer las propias (García, 2001).

En la Agenda 21 para el sector de viajes y turismo (1995) y en la Carta del turismo sostenible de Lanzarote (1995), se indica que el desarrollo turístico debe ser soportable ecológicamente, viable económicamente $\mathrm{y}$ equitativo desde una perspectiva ética y social para las comunidades locales.

Se afirma que los organismos competentes deben promover la planificación integrada del turismo como contribución al desarrollo sostenible; fomentando programas con acciones positivas y preventivas que permitan observar los logros alcanzados, informar de los resultados e intercambiar experiencias; para lo cual hay que apoyar e impulsar sistemas de gestión turística ambientalmente compatibles (Robert, 2010).

Intentar avanzar hacia la sostenibilidad puede no tener sentido sin alguna forma objetiva de evaluarla en función de respetar los principios fundamentales que la definen, de ahí la importancia de disponer de herramientas de evaluación que son un componente central de los procesos de planificación y de gestión del turismo.

La comunidad científica a nivel internacional, promueve investigaciones encaminadas al desarrollo sostenible y a la sostenibilidad turística, significando los mayores avances hacia la definición de instrumentos y herramientas para su evaluación. (Torres, 2016; Um, 2010 Ramos y Caeiro, 2010; Pulido y Sánchez, 2009; Pérez y Nel-lo, 2009; Pérez et. al, 2009; Blancas et al., 2009 Martínez, López, e Santos, 2007; Choi y Sirikaya, 2005).

Los mismos aun evidencian carencias o vacíos para evaluar las dinámicas complejas que concurren en las interrelaciones de un destino turístico. Estas interrelaciones se manifiestan en todas las fases del proceso de gestión del mismo, lo que deviene en la complejidad del diagnóstico o evaluación de los avances hacia la sostenibilidad o no, en aras de tomar decisiones pertinentes en función de los objetivos de desarrollo determinados.

Para evaluar la sostenibilidad turística, tradicionalmente se utilizan tres dimensiones: ambiental, económico y social (este último se ha unido con el cultural y se aborda como socio-cultural), a las que se le han incorporado otras como la político-institucional (Yasarata et al., 2009; Márquez y Cuétara, 2007), espaciales (van den Berg y Verbruggen, 1998) y 
tecnológico (Crespo, 2011; Fortuny et al., 2008), aunque menos considerado en las evaluaciones que se realizan.

\section{Marco metodológico.}

La investigación es de tipo descriptiva, pues como su tipología lo indica en el desarrollo del estudio científico se describe lo más preciso posible el fenómeno que se investiga (Perelló, 2014). Al mismo tiempo Hernández, Fernández \& Baptista, (2006) plantean que la investigación científica es un conjunto de procesos sistemáticos y empíricos que se aplican al estudio de un fenómeno; es dinámica, cambiante y evolutiva; específicamente, la investigación turística está concebida como la formulación de preguntas, la sistemática colección de información para responder a esas preguntas y la organización y análisis de los datos con el fin de obtener pautas de comportamiento, relaciones y tendencias que ayuden al entendimiento del sistema, a la toma de decisiones o a la construcción de predicciones bajo el abanico de varios escenarios alternativos de futuro (Sancho, 2008).

Siguiendo a Cerezal y Fiallo (2002), citados por González (2014), la experiencia afirma que en toda investigación se integran lo cualitativo con lo cuantitativo, lo cual se aplica a este trabajo.

Se determina el comportamiento de las herramientas métodos y modelos para evaluar la sostenibilidad que reconoce la literatura científica consultada. El proceso metodológico se desarrolló en 3 etapas, las cuales se caracterizan a continuación:

Etapa preparatoria: el completamiento de las bases teóricas se basó en el análisis bibliográfico como método empírico, que permitió la identificación y consulta de fuentes de información necesarias que permitieron extraer y recopilar datos esenciales para la conformación de la investigación.

Los principales tópicos de consulta desde la bibliografía especializada en los temas de sostenibilidad, y de gestión de destinos turísticos. La utilización de este método permitió reconocer las prácticas más generalizadas de herramientas modelos y métodos para evaluar la sostenibilidad en destinos turísticos.

Etapa trabajo de mesa: como principal técnica de recopilación de información en esta etapa partió de una amplia revisión bibliográfica especializada sobre el tema como se refiere en la etapa anterior, que permitió realizar una comparación de herramientas modelos y métodos que reconoce la literatura científica como los más utilizados, siguiendo los parámetros de comparación declarados por Torres (2016): Enfoque Integrado, determinación de equilibrio, establece relaciones complejas entre elementos o variable, compara resultado con objetivos de sostenibilidad, establece áreas de mejora, determina estado de sostenibilidad general y analiza la tendencia. Se analizó el comportamiento por cada parámetro desde un análisis 
matemático expresando en porcientos la cantidad de parámetros para cada herramienta, modelo y método.

En un segundo momento de esta etapa se realiza un repaso, a los indicadores por ser la herramienta que ha demostrado una mayor utilización en la evaluación de la sostenibilidad de los destinos para ellos se tomaron las propuesta que reconoce la literatura científica tanto internacional y nacional como las más extendidas y generalizadas Asociación de Estados del Caribe (AEC) por su nombre en español (1999), el Ministerio de Turismo de Cuba(MINTUR)de conjunto con el Ministerio de Tecnología y Medio Ambiente(CITMA)(2003),la Organización Mundial del Turismo(OMT)(2005) la Unión Europea.

Etapa final: se organizó y procesó la información obtenida, lo cual condujo al análisis de resultados, el cual responde al objetivo general planteado en la investigación enfocado sobre la base de determinar herramienta, modelo y método que permitan evaluar la sostenibilidad de un destino turístico.

\section{Análisis de los Resultados.}

Por la complejidad que implica la evaluación de la sostenibilidad en los destinos turísticos, se realiza un análisis de las herramientas, modelos y métodos más utilizados y puede concluirse que (tabla1):

- Las herramientas y métodos permiten realizar enfoques integrados, no así los modelos, sin embargo, de manera general (las herramientas, métodos y modelos) no determinan estado de equilibrio, ni estado de sostenibilidad general.

- Solamente los sistemas de indicadores (herramientas) son los que permiten comparar los resultados obtenidos con los objetivos de la sostenibilidad no comportándose de la misma manera los (métodos) que lo logran desde: el índice de sostenibilidad e indicadores compuestos y en el caso de los (modelos) exhiben un mejor comportamiento: análisis de la huella ecológica, AMOEBA, panel de la sostenibilidad, barómetro de la sostenibilidad.

- Mayoritariamente (las herramientas, métodos y modelos) no permiten establecer relaciones complejas entre elementos o variables, a excepción del razonamiento cualitativo (herramienta), el análisis emergético (modelo), el sistema-entorno y sistemas de cuentas medioambientales y económicas (métodos). Además, de modo general no permiten realizar análisis de tendencia sin embargo en todos los casos se establecen áreas de mejora, que permiten una retroalimentación en la búsqueda de un buen desempeño y de buenas prácticas en función de los objetivos del destino. 
Tabla 1. Comportamiento de los parámetros en función de las herramientas, métodos y modelos.

\begin{tabular}{|c|c|c|c|c|c|c|c|c|}
\hline & $\begin{array}{l}\text { Enfoque } \\
\text { Integrado }\end{array}$ & $\begin{array}{c}\text { Determinació } \\
\text { n de } \\
\text { Equilibrio }\end{array}$ & $\begin{array}{l}\text { Establece } \\
\text { relaciones } \\
\text { complejas } \\
\text { entre } \\
\text { elementos } \\
\text { o variable }\end{array}$ & $\begin{array}{c}\text { Compara } \\
\text { resultado } \\
\text { con } \\
\text { objetivos } \\
\text { de } \\
\text { sostenibilid } \\
\text { ad } \\
\end{array}$ & $\begin{array}{c}\text { Establece } \\
\text { áreas de } \\
\text { mejora }\end{array}$ & $\begin{array}{l}\text { Determina } \\
\text { estado de } \\
\text { sostenibilida } \\
\text { d general }\end{array}$ & $\begin{array}{l}\text { Analiza la } \\
\text { tendencia }\end{array}$ & Total \\
\hline Herramientas & $100 \%$ & $25 \%$ & $25 \%$ & $25 \%$ & $100 \%$ & $75 \%$ & $25 \%$ & 4 \\
\hline Métodos & $100 \%$ & $33,3 \%$ & $33,3 \%$ & $66,6 \%$ & $100 \%$ & $100 \%$ & $33,3 \%$ & 3 \\
\hline Modelos & $33,3 \%$ & $8,3 \%$ & $16,6 \%$ & $33,3 \%$ & $100 \%$ & $8,3 \%$ & $16,6 \%$ & 12 \\
\hline
\end{tabular}

Fuente: Elaboración propia.

Aunque es significativo, que en los últimos años el esfuerzo se ha focalizado en la búsqueda de herramientas apropiadas varias son las organizaciones nacionales e internacionales que generan iniciativas en la concepción de guías que sirvan de apoyo en la construcción y selección de los mismos; entre las más generalizadas y extendidas se encuentran: la Organización Mundial del Turismo (OMT, 2005), la Unión Europea (2016), Asociación de Estados del Caribe (AEC, 1999), el Ministerio de Turismo de Cuba de conjunto con el Ministerio de Tecnología y Medio Ambiente (CITMA-MINTUR,2003); propuestas que serán analizadas a continuación:

Resumen del análisis comparado de las propuestas de indicadores.

Análisis de la propuesta de indicadores planteada por la Asociación de Estados del Caribe (AEC), 1999.

La Asociación de Estados del Caribe propone un conjunto de indicadores para armonizar los criterios de categorización para la inclusión de esos destinos en la Zona de Turismo Sostenible del Caribe, los cuales fueron identificados y concertados a través de los trabajos del Grupo de Expertos en Indicadores de Sostenibilidad del Comité Especial de Turismo Sostenible de la AEC.

La propuesta presenta 14 indicadores con una mayor concentración de los mismos en la dimensión ambiental (ocho), sociocultural (cuatro) y económica (dos).

Análisis de la propuesta de indicadores planteada por el Ministerio de Turismo de Cuba (MINTUR) en conjunto con el Ministerio de Ciencia, Tecnología y Medio Ambiente de Cuba (CITMA), 2003.

La propuesta de indicadores para el destino Cuba, la cual abarca todos los indicadores de sostenibilidad turística de la (AEC), los criterios de selección estuvieron basados en las 
características del área, así como los intereses de los gestores y los decisores (por lo que exceptúa el de prostitución infantil ya que no responde a las problemáticas de Cuba) e incorpora otros 16 que resultan de importancia para el logro de los objetivos y metas del desarrollo turístico en el país.

Esta selección comprende un total de 29 indicadores, es muy general y poco específica para los destinos turísticos cubanos.

En ellos, hay una mayor presencia de la dimensión ambiental (17 indicadores representando el $61 \%$ del total) con respecto a la económica (9 indicadores $22.5 \%$ ) y la sociocultural (8 indicadores $16.5 \%$ ).

\section{Análisis de la propuesta planteada en la Guía Práctica de Indicadores de Desarrollo Sostenible para los Destinos Turísticos de la OMT (2005).}

Esta propuesta ofrece elementos y referencias para elaborar indicadores que contribuyan a atenuar los problemas o dificultades en materia de política y gestión que puedan encontrarse en los diferentes destinos, por lo que no constituye una fórmula de indicadores, sino que está encaminada a generar ideas que promuevan propuestas.

En total se examinan 13 cuestiones generales que abarcan en su conjunto 42 comunes específicas, que a vez su vez responden a 231 componentes y a una propuesta de 750 indicadores.

El análisis permitió conocer además que predominan las cuestiones e indicadores pertenecientes a la dimensión sociocultural por encima de la ambiental y la económica, destacándose como elemento notorio que la cuestión seis (Aprovechamiento de los beneficios económicos del turismo) es la que presenta el mayor número de cuestiones específicas (siete), de componentes (34) y de indicadores (153).

Esta guía presenta además una selección de las cuestiones básicas (12) e indicadores básicos (29) De las cuestiones básicas predominan las que responden a la dimensión ambiental con un total de seis, para 14 indicadores, lo que representa el 50\% del total.

\section{Propuesta de indicadores de sostenibilidad planteada por la Unión Europea, 2016.}

El Sistema de Indicadores del turismo europeo (ETIS), por sus siglas en inglés pretende ayudar a los destinos a monitorear y medir el desarrollo del turismo sostenible.

El mismo propone un total de 37 indicadores divididos en cuatro categorías: gestión del destino (tres indicadores), valor económico (nueve indicadores), impacto social y cultural (10 indicadores) e impacto ambiental (15 indicadores).

Para una mejor comprensión de los resultados se muestra el gráfico 1 que resume el comportamiento general de las propuestas analizadas. 
Gráfico 1: Comportamiento por dimensiones por cada propuesta.

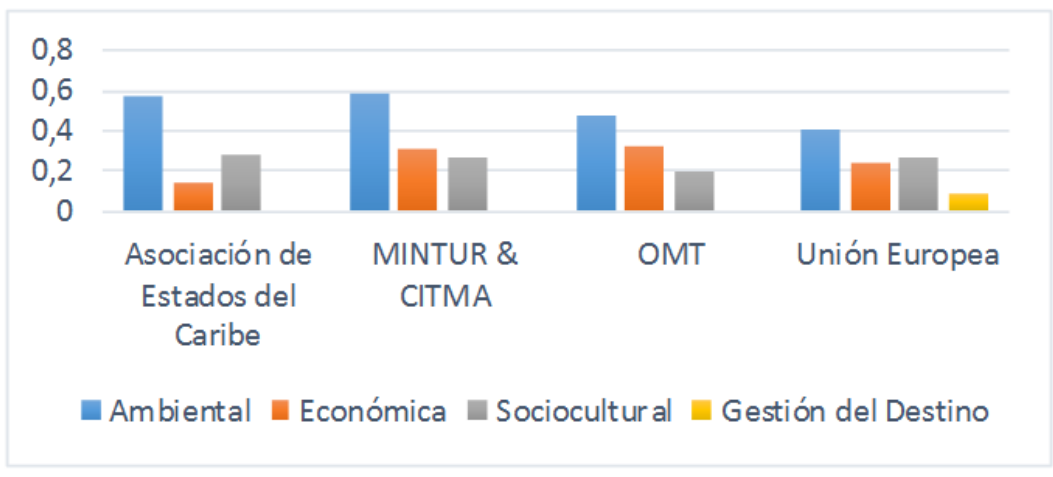

Fuente: Elaboración Propia.

Entre los últimos estudios que se realizan en Cuba en la búsqueda de herramientas para evaluar la sostenibilidad de los destinos hay que destacar los desarrollados desde la Facultad de Turismo de conjunto con el MINTUR Torres (2016); Robert y Blanco (2016); Echarri y Delis (2015) los cuales plantean como parte de sus resultados que los indicadores son la herramienta más utilizada en el presente y probablemente lo seguirán siendo en el futuro preferiblemente en combinación con otro instrumento, al mismo tiempo Robert y Blanco (2016) aseveran que las propuestas nacen de fuentes gubernamentales que se centra específicamente en lograr una propuesta de indicadores para evaluar la sostenibilidad del turismo del destino Cuba a partir de la disponibilidad de datos desde fuentes oficiales de acceso público.

El estudio partió del análisis de la propuesta realizada por CITMA-MINTUR (2003) y la OMT (2005) contrastando las coincidencias de indicadores con la información que se dispone en la ONEI como única fuente gubernamental de acceso público en Cuba permitiendo arribar a una nueva propuesta de indicadores para evaluar el destino Cuba presentando las siguientes características (Robert y Blanco, 2016):

- La propuesta tiene un total de 22 indicadores, 11 perteneciendo a la dimensión económica, cuatro a la dimensión ambiental y siete a la social, reflejando un desequilibrio entre las dimensiones de la sostenibilidad.

- No existen una continuidad en las series de datos.

- Cuando se refiere al nivel territorial que comprende el indicador y este es provincial o municipal, solamente se recogen los datos referidos a la provincia de La Habana y a sus municipios.

\section{Discusión.}


De modo general las herramientas, métodos y modelos no responden al análisis de la sostenibilidad de un destino turístico según los parámetros seleccionados, respondiendo solo al $100 \%$ en el caso de establecer áreas de mejora, lo que denota las limitaciones de los mismos y los vacíos que quedan para evaluar la sostenibilidad de un destino, partiendo de que coexisten dos grandes complejidades el de llevar a la práctica el concepto de la sostenibilidad y el de la gestión de un destino con todos los procesos que en él se producen. Aunque es significativo este comportamiento positivo de todos en responder a establecer áreas de mejora si se tiene en cuenta que permiten una retroalimentación en la búsqueda de un buen desempeño y de aplicar buenas prácticas en función de los objetivos del destino.

Siguiendo una misma lógica de discusión la primera propuesta de indicadores (AEC) presenta un desequilibrio marcado entre las dimensiones, lo que limita la relación específica causa/efecto entre el turismo y las diferentes dimensiones de la sostenibilidad.

La propuesta de Cuba, aunque más condensada denota la falta de equilibrio entre las dimensiones, no es capaz de medir el nivel de la sostenibilidad del turismo en los diferentes destinos del país y no permite una comparación entre destinos y en el tiempo, dificultando monitorear el comportamiento de la actividad turística determinado fundamentalmente por la falta de información.

La OMT por su parte desde su propuesta tiene un enfoque más abarcador en tanto asume un mayor número de cuestiones a evaluar en las diferentes dimensiones, facilitando su adecuación a diferentes tipos de espacios turísticos y recomendando un conjunto mínimo para realizar la descripción del estado de un destino, aunque no permite realizar análisis más complejos, si logra comparar comportamientos entre ellos; pero evidenciando desequilibrio a la hora de evaluar las relaciones inter e intradimensionales de la sostenibilidad en un destino turístico.

La Unión Europea limita el análisis holístico del destino con la selección de tres indicadores dentro de la categoría gestión del destino, sin embargo, es de considerar que los indicadores son seleccionados para ser parte integrante de los procesos de planificación, gestión y monitoreo, se debe partir del criterio de que entre ellos se producen relaciones intradimensionales tratándose de interacciones y de enfoques transectoriales, transdiciplinarios y transversales.

La propuesta más reciente de indicadores para el destino Cuba realizada por (Robert y Blanco, 2016), aun cuando su comportamiento difiere del que evidenciaron el resto de las propuesta analizadas, donde la dimensión ambiental era la que ocupaba el mayor número de indicadores, en este caso ocupado por la dimensión económica, de igual manera presenta un desequilibrio entre las dimensiones con la incapacidad de responder al enfoque holístico que exige la evaluación de la sostenibilidad en un destino turístico. 
En función del análisis realizado debe considerarse como un elemento determinante en la evaluación de la sostenibilidad de los destinos turísticos la selección de los indicadores, ya que la misma puede estar condicionada por la percepción de quienes construyen los indicadores lo que puede traer aparejada subjetividad, impactos que pueden quedar solapados así como una visión unidimensional de la misma; si se tiene en cuenta que la evaluación de la sostenibilidad no puede significar solo cuidar ecosistemas y recursos, sino que debe conducir el desarrollo equilibrado de los componentes medioambientales, socioculturales y económicos.

\section{Conclusiones.}

- Se pudo determinar de manera general que las prácticas más generalizadas (herramientas, modelos y métodos) para evaluar la sostenibilidad aun no logran el enfoque multidimensional de las dinámicas complejas que concurren en un destino, lo que evidencia la necesidad de realizar investigaciones en la búsqueda de nuevos instrumento desde otras áreas del conocimiento dirigidos a reconocer y a comprender la visión holística de la sostenibilidad en los destinos turísticos y que al mismo tiempo disminuyan el carácter subjetivo de las variables que integran las dimensiones de la sostenibilidad.

- Si bien el estudio demuestra que los indicadores son la herramienta más utilizada hasta hoy para evaluar la sostenibilidad de los destinos, el análisis comparativo de las propuestas reconoce las limitaciones que presentan, demostrando la falta de equilibrio entre las dimensiones, impidiendo determinar niveles de sostenibilidad en los destinos turísticos, así como comparar comportamientos espaciales y temporales.

\section{Referencias bibliográficas.}

Ávila Bercial, R., \& Barrado Timón, D. (2005). Nuevas tendencias en el desarrollo de destinos turísticos: marcos conceptuales y operativos para su planificación y gestión. Cuadernos de Turismo, 15, 27-43

Asociación de Estados del Caribe. (1999) "Declaración para el establecimiento de la zona de turismo sustentable del Caribe, II Cumbre de Jefes de Estado y/o Gobierno de la A.E.C.”, Santo Domingo.

CITMA - MINTUR. (2003) "Indicadores de sostenibilidad para el turismo en Cuba", Documento preliminar, La Habana.

Cerezal, J.; Fiallo, J. (2002) Los métodos científicos en las investigaciones pedagógicas. Habana. 
Echarri M, Delis,C (2015), "Evaluación de indicadores de sostenibilidad turística para el Centro Histórico de La Habana en el período 2010-2014” Trabajo de Diploma. Facultad de Turismo, Universidad de La Habana.

Echarri, M. (2006) Análisis geográfico del turismo en ciudades patrimoniales. Caso de estudio: Centro Histórico de La Habana. Tesis Doctoral. Facultad de Geografía, Universidad de La Habana, La Habana, Cuba.

European Commission. (2016) “The European Tourism Indicator System. ETIS toolkit for sustainable destination managment", Disponible en: http://ec.europa.eu/growth/sectors/tourism/offer/sustainable/indicators/index_en.htm

Fortuny J; LL., Cuatrecasas; O. ,Cuatrecasas; J. ,olivella (2008) Metodología de implantación de la gestión lean en plantas industriales, Universidad Politécnica de Catalunya, 14 pp.

García, G. "Gestión del desarrollo sostenible en turismo", Publicaciones CODIMA, Barcelona, 2001

Hernández, R.; Fernández, C. y Batista, P. (2000). Metodología de la investigación. (2da. ed.). Editorial McGraw-Hill Interamericana. D.F. Méjico.

Márquez, L., \& Cuétara, L, 2007, Propuesta y aplicación de un sistema de indicadores para determinar el índice de desarrollo sostenible global (IDSG) de un destino turístico, Patameno, Venezuela.

Organización de las Naciones Unidas (1987). Nuestro Futuro Común o Informe Brutland.. Disponible en: http://www.tij.uia.mx/elbordo/vol05/. (consulta: octubre del 2019).

Organización Mundial del Turismo (OMT) (1995) “Carta de Turismo sostenible, aprobada en la Conferencia Mundial sobre Turismo Sostenible realizada en Lanzarote”, Islas Canarias.

Organización Mundial del Turismo (OMT) (1999) Agenda para planificadores locales: turismo sostenible y gestión municipal: edición para América latina y el Caribe. Madrid, España.

Organización Mundial del Turismo (OMT). (2004) “Gestión de la saturación turística en sitios de interés natural y cultural. Guía práctica”, España.

Organización Mundial del Turismo (OMT) (2005) Indicadores de desarrollo sostenible para los destinos turísticos. Guía práctica. Madrid, España. 
Organización Mundial del Turismo (OMT) (2006) Programa de las Naciones Unidas para el Medio Ambiente (PNUMA). Por un turismo más sostenible. Guía para responsables políticos. Madrid, España.

Organización Mundial del Turismo (OMT) (2017) Panorama OMT del turismo internacional.

Perelló, J. 2014 "La edición y publicación de los resultados de la investigación” Clase Metodológica Instructiva. Facultad de Turismo. Universidad de La Habana.

Robert, M.O. y Blanco, A. (2016). Propuesta de indicadores para evaluar la sustentabilidad turística del destino Cuba. Trabajo de Diploma. Universidad de la Habana. Facultad de Turismo. La Habana. Cuba

Robert, M. O. 2010 Evaluación de indicadores de sustentabilidad turística para el centro Histórico de La Habana en el período 2000-2008. Tesis presentada en opción al título de Master en Gestión Turística. Ciudad de La Habana, Cuba: Facultad de Turismo. Universidad de La Habana.

Silveira, M. A. Turismo y sustentabilidad. Entre el discurso y la acción. (U. f. Panamá, Ed.) Estudios y perspectivas en Turismo, Vol.14. 2005.

Tarlombani, M. A. (2005) "Turismo Y Sustentabilidad: Entre el discurso y la acción”. Universidad Federal de Paraná Curitiba. Brasil.

Torres, L (2016). "Propuesta de esquema metodológico para la evaluación de la sostenibilidad del desarrollo turístico de destinos caso La Habana” Tesis Doctoral. Universidad de La Habana, Cuba.

Vera, J. F. (Coord.), López Palomeque, F., Marchena, M., \& Anton, S. (2013). Análisis territorial del turismo y planificación de destinos turísticos. Valencia: Tirant Humanidades.

\section{Ciencia}




\section{Para citar el artículo indexado}

Robert Beatón, M. O., Echarri Chávez, M., Díaz Saraiba, J., \& Martínez Robert, V. (2020). La sostenibilidad: una mirada hacia las principales herramientas de evaluación en los destinos turísticos. AlfaPublicaciones, 1(3), 39-52. https://doi.org/10.33262/ap.v1i3.3

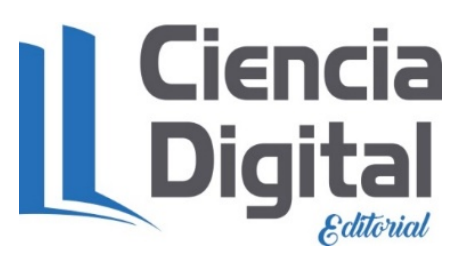

El artículo que se publica es de exclusiva responsabilidad de los autores y no necesariamente reflejan el pensamiento de la Revista Alpha Publicaciones.

El artículo queda en propiedad de la revista y, por tanto, su publicación parcial y/o total en otro medio tiene que ser autorizado por el director de la Revista Alpha Publicaciones.
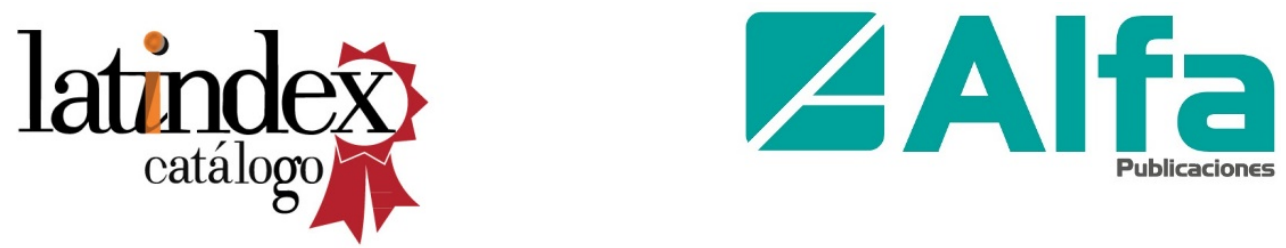